Marc Leone

Carole Bechis

Karine Baumstarck

Jean-Yves Lefrant

Jacques Albanèse

Samir Jaber

Alain Lepape

Jean-Michel Constantin

Laurent Papazian

Nicolas Bruder

Bernard Allaouchiche

Karine Bézulier

François Antonini

Julien Textoris

Claude Martin

For the AZUREA Network Investigators

\section{Erratum to: De-escalation versus continuation of empirical antimicrobial treatment in severe sepsis: a multicenter non-blinded randomized noninferiority trial}

Published online: 7 October 2014

(C) Springer-Verlag Berlin Heidelberg and ESICM 2014

The online version of the original article can be found under doi:10.1007/s00134-014-3411-8.

M. Leone ( $\bullet$ - C. Bechis · F. Antonini - C. Martin Service d'anesthésie et de réanimation, Hôpital Nord, Chemin des Bourrely, 13015 Marseille, France

e-mail: marc.leone@ap-hm.fr

Tel.: +33491968650

M. Leone $\cdot$ L. Papazian · J. Textoris

Unité de Recherche sur les Maladies Infectieuses et Tropicales

Émergentes, Centre National de la Recherche Scientifique-Unité

Mixte de Recherche 7278, Aix Marseille Université,

Marseille, France

K. Baumstarck

Unité d'Aide Méthodologique à la Recherche Clinique et

Epidémiologique, Aix Marseille Université, Marseille, France

J.-Y. Lefrant

Service des Réanimations Pôle Anesthésie Réanimation Douleur

Urgence, CHU Nîmes, Nîmes, France

J. Albanèse

Hôpital La Conception, Marseille, France

S. Jaber

Hôpital Saint-Eloi, Montpellier, France
A. Lepape

Hôpitaux Lyon Sud, Lyon, France

J.-M. Constantin

Réanimation polyvalente, Hôpital universitaire Estaing, ClermontFerrant, France

L. Papazian

Hôpital Nord, Marseille, France

N. Bruder

Hôpital la Timone, Marseille, France

B. Allaouchiche

Hôpital Edouard Herriot, Lyon, France

K. Bézulier

Centre d'Investigation Clinique 9502, Aix Marseille Université, Assistance Publique Hôpitaux de Marseille, Marseille, France

\section{Erratum to: Intensive Care Med (2014) 40:1399-1408 DOI 10.1007/s00134-014-3411-8}

The authors' conclusion about ICU length of stay did not reflect their data. It is more correct to conclude that the length of stay was not different between the de-escalation strategy and the continuation strategy. The authors apologize for this error and for any inconvenience caused. 